\title{
UTILIZAÇÃO DE PLANTAS MEDICINAIS POR PACIENTES HIPERTENSOS E DIABÉTICOS: ESTUDO TRANSVERSAL NO NORDESTE BRASILEIRO
}

\section{Use of medicinal plants by patients with hypertension and diabetes: a cross- sectional study in Northeastern Brazil}

\section{Utilización de plantas medicinales por pacientes hipertensos y diabéticos: estudio transversal en el Noreste brasileño}

\section{Taís Batista Virgínio}

Universidade de Fortaleza - UNIFOR - Fortaleza (CE) - Brasil

Karla Soares de Castro

Universidade de Fortaleza - UNIFOR - Fortaleza (CE) - Brasil

Ana Luiza Almeida de Lima

Universidade Estadual do Ceará - UECE - Fortaleza (CE) - Brasil

Juliana Vieira Rocha

Universidade de Fortaleza - UNIFOR - Fortaleza (CE) - Brasil

Isabela Melo Bonfim

Universidade de Fortaleza - UNIFOR - Fortaleza (CE) - Brasil

Adriana Rolim Campos

Universidade de Fortaleza - UNIFOR - Fortaleza (CE) - Brasil

\section{RESUMO}

Objetivo: Descrever a utilização de plantas medicinais por pacientes hipertensos e/ou diabéticos atendidos em unidades de atenção primária à saúde e em um ambulatório especializado. Métodos: Pesquisa quantitativa, descritiva, exploratória e transversal, realizada em 16 Unidades de Atenção Primária à Saúde da Regional VI e em um ambulatório especializado do Hospital Universitário Walter Cantídio, em Fortaleza, Ceará, Brasil, de abril de 2014 a janeiro de 2015, com 122 participantes. Realizou-se uma entrevista, na qual foi aplicado um formulário composto por 41 perguntas, distribuídas em três partes: dados sociodemográficos; dados sobre a patologia, tratamento e adesão medicamentosa convencional; e dados sobre uso de plantas medicinais. A análise descritiva e inferencial dos dados partiu do programa Statistical Package for the Social Sciences, versão 20.0. Resultados: Dos 122 participantes, 66 (54,09\%) eram do nível terciário de atenção, 50,82\% eram maiores de 60 anos ( $n=62), 70,49 \%$ eram mulheres $(n=86), 31,97 \%$ apresentavam ensino fundamental incompleto $(n=39), 45,08 \%$ eram aposentados/pensionistas $(n=55), 55,74 \%$ viviam com companheiro $(n=68)$ e $32,79 \%$ eram hipertensos/diabéticos $(n=40)$. Verificou-se associação significativa entre uso de plantas e nível de atenção ( $p=0,0001)$, escolaridade $(p=0,021)$ e patologia de base $(p=0,0001)$. Conclusão: $O$ uso de plantas medicinais se faz presente na população pesquisada e a elevada prevalência da utilização requer mais estudos acerca dos benefícios e malefícios dessa prática, bem como a capacitação dos profissionais envolvidos, com consequente segurança, eficácia e consumo racional pelos pacientes.

Descritores: Plantas medicinais; Hipertensão; Diabetes Mellitus.

\section{ABSTRACT}

Objective: To describe the use of medicinal plants by hypertensive and/or diabetic patients served by primary health care centers and at a specialized outpatient clinic. Methods: A quantitative, descriptive, exploratory and cross-sectional study was carried out with 122 participants in 16 Primary Health Care Centers run by the VI Regional Executive Secretariat and at a specialized outpatient clinic of the Walter Cantídio Teaching Hospital in Fortaleza, Ceará, Brazil, from April 2014 to January 2015. Interviews were carried out using a form with 41 questions distributed into three sections: sociodemographic data; data on the disease,

Este artigo foi selecionado, corrigido e aprovado para publicação pelo VI Seminário Internacional em Promoção da Saúde, seguindo suas normas e formatação. 
treatment and conventional medication adherence; and data on the use of medicinal plants. Descriptive and inferential analysis of data was performed using the Statistical Package for the Social Sciences version 20.0. Results: Of the 122 patients, 66 $(54.09 \%)$ received tertiary care, $50.82 \%$ were older than 60 years $(n=62), 70.49 \%$ were women $(n=86), 31.97 \%$ had incomplete primary education ( $n=39), 45.08 \%$ were retired/pensioners $(n=55), 55.74 \%$ lived with a partner $(n=68)$ and $32.79 \%$ were hypertensive/ diabetics $(n=40)$. Use of plants was significantly associated with level of care $(p=0.0001)$, education $(p=0.021)$ and underlying disease $(p=0.0001)$. Conclusion: The use of medicinal plants was present in the study population and the high prevalence of the use points to the need of further studies about the benefits and harms of this practice, the training of the professionals involved, and, consequently, the safety, efficacy, and rational use by the patients.

Descriptors: Medicinal Plants; Hypertension; Diabetes Mellitus.

\section{RESUMEN}

Objetivo: Describir la utilización de plantas medicinales por pacientes hipertensos y/o diabéticos asistidos en unidades de atención primaria de salud y ambulatorio especializado. Métodos: Investigación cuantitativa, descriptiva, exploratoria y transversal realizada en 16 Unidades de Atención Primaria de Salud de la Regional VI y en un ambulatorio especializado del Hospital Universitario Walter Cantídio de Fortaleza, Ceará, Brasil, entre abril de 2014 y enero de 2015 con 122 participantes. Se realizó una entrevista en la cual se aplicó un formulario con 41 preguntas distribuidas en tres partes: los datos sociodemográficos; los datos sobre la patología, el tratamiento y la adhesión medicamentosa convencional; y los datos sobre el uso de plantas medicinales. Para el análisis descriptivo e inferencial de los datos se utilizó la versión 20.0 del programa Statistical Package for the Social Sciences. Resultados: Entre los 122 participantes, 66 (54,09\%) eran del nivel terciario de atención, el 50,82\% tenían más de 60 años ( $n=62)$, el 70,49\% eran mujeres ( $n=86)$, el 31,97\% tenían la educación primaria incompleta $(n=39)$, el $45,08 \%$ eran jubilados/pensionistas $(n=55)$, el $55,74 \%$ vivian con compañero $(n=68)$ y el $32,79 \%$ eran hipertensos/diabéticos $(n=40)$. Se verificó una asociación significativa entre el uso de plantas y el nivel de atención ( $p=0,0001)$, la escolaridad $(p=0,021)$ y la patología de base $(p=0,0001)$. Conclusión: El uso de plantas medicinales está presente en la población investigada y la elevada prevalencia de su utilización requiere más estudios sobre los beneficios y maleficios de esa práctica así como la capacitación de los profesionales involucrados, con consecuente seguridad, eficacia y consumo racional de parte de los pacientes.

Descriptores: Plantas Medicinales; Hipertensión; Diabetes Mellitus.

\section{INTRODUÇÃO}

O uso das terapias alternativas e complementares apresenta ampla diversidade no mundo, conforme as características culturais e econômicas de cada país. Segundo a Organização Mundial da Saúde (OMS), nos países em desenvolvimento, a medicina tradicional é a única abordagem de cuidado à saúde disponibilizada para a imensa maioria da população. Diferentemente, nos países economicamente desenvolvidos, as terapias alternativas e complementares são utilizadas de acordo com as crenças dos cidadãos ${ }^{(1)}$.

No Brasil, por meio de iniciativas do Ministério da Saúde (MS), a utilização de plantas medicinais e de fitoterápicos tem sido incentivada. No ano de 2006, foi publicada a Portaria n. 971, que traz como proposta de opção terapêutica no Sistema Único de Saúde (SUS) a Política Nacional de Práticas Integrativas e Complementares (PNPIC), que inclui as plantas medicinais, a fitoterapia, a homeopatia, dentre outras práticas ${ }^{(2)}$.

No estado do Ceará, a iniciativa foi dada por ocasião do Decreto n. 30.016, de 30 de dezembro de 2009, que regulamentava a Lei Estadual n. 12.951, de 7 de outubro de 1999. A referida lei tratava sobre a política de implantação da fitoterapia em Saúde Pública no estado do Ceará. Embora alguns estados tenham se antecipado em relação à legislação federal, é o documento da PNPIC que, atualmente, referencia estados e municípios na formulação de políticas voltadas ao assunto $^{(3)}$.

Dentre as doenças crônicas, a diabetes mellitus (DM) e a hipertensão arterial sistêmica (HAS) estão entre as mais prevalentes, e seu tratamento e controle requerem mudanças de hábitos e estilo de vida no que se refere à dieta, ingestão de medicamentos e prática de atividade física. No entanto, aparentemente, existem poucos estudos discutindo o uso de plantas medicinais por pacientes com DM e HAS ${ }^{(4)}$.

O uso de plantas medicinais é, equivocadamente, entendido pela população, de uma maneira geral, como o emprego de fitoterapia. Segundo conceito da Agência Nacional de Vigilância Sanitária (ANVISA), o medicamento fitoterápico é aquele obtido através do emprego exclusivo de matérias-primas vegetais, sendo caracterizado pelo conhecimento da eficácia e dos riscos de seu uso, assim como pela reprodutibilidade e constância de sua qualidade. São validadas sua eficácia e segurança através de levantamentos etnofarmacológicos de utilização e de documentações tecnocientíficas em publicações ou ensaios clínicos fase $3^{(5)}$. 
Apesar de já existirem diversos estudos relativos ao uso, toxicidade e eficácia das plantas medicinais, as produções científicas ainda são precárias no que se referem ao modo como os vegetais estão sendo consumidos, quais os benefícios e como se poderá capacitar e habilitar os profissionais de saúde para orientação e aconselhamento da utilização como opção terapêutica no SUS.

Dessa forma, o objetivo da presente pesquisa foi descrever a utilização de plantas medicinais por pacientes hipertensos e/ou diabéticos atendidos em unidades de atenção primária à saúde em um ambulatório especializado no município de Fortaleza.

\section{MÉTODOS}

Trata-se de estudo com abordagem quantitativa, descritiva, exploratória e transversal, realizado em 16 Unidades de Atenção Primária à Saúde (UAPS) e em um ambulatório especializado pertencente ao Hospital Universitário Walter Cantídio, da Universidade Federal do Ceará, ambos integrados ao SUS e sediados no município de Fortaleza, Ceará, Brasil, no período de abril de 2014 a janeiro de 2015.

De uma amostra pretendida de 210 hipertensos e/ou diabéticos que participaram da coleta de dados durante o período do estudo, somente 122 formulários foram considerados válidos. Houve extravio de 49 formulários, 33 participantes não preencheram critérios de inclusão, e seis se recusaram a continuar após a conclusão da coleta.

Consideraram-se critérios de inclusão: pacientes com diagnóstico clínico-laboratorial, há pelo menos seis meses de HAS, independente do estágio (1, 2, 3 ouhipertensão sistólica isolada), de acordo com os critérios da Sociedade Brasileira de Hipertensão e/ou DM, independentemente da sua classificação (tipo 1 ou 2), de acordo com a Sociedade Brasileira de Diabetes; aqueles maiores de 18 anos; de ambos os sexos e que buscaram atendimentos nas UAPS da regional VI e no ambulatório especializado citado no período do estudo.

Consideraram-se os seguintes critérios de exclusão: pacientes portadores de diabetes gestacional e aqueles com distúrbios psíquicos e neurológicos que limitassem a compreensão adequada das informações.

Após conhecimento dos dias e horários destinados para atendimento dos pacientes hipertensos e/ou diabéticos de cada serviço citado, a pesquisadora ou o bolsista se dirigiam aos pacientes que aguardavam atendimento multiprofissional nas salas de espera, convidando-os, aleatoriamente, a participar do estudo, e fornecendo esclarecimentos quanto à forma de condução da pesquisa. Depois de explicado o contexto da investigação, aqueles que aceitaram participar e assinaram o Termo de Consentimento Livre e Esclarecido (TCLE) foram recrutados para a aplicação do formulário.

As entrevistas aconteceram em consultórios destinados para fins de consulta de enfermagem, conforme a estrutura física e cronograma de cada serviço. A técnica adotada foi uma entrevista estruturada, com aplicação de um formulário estruturado contendo perguntas subjetivas e objetivas. As informações foram registradas no formulário pelo entrevistador e o contato com cada entrevistado se deu em um único momento.

O formulário era composto por 41 perguntas, distribuídas em três partes: dados sociodemográficos; dados sobre a patologia, tratamento e adesão medicamentosa convencional; e dados sobre uso de plantas medicinais.

Para análise, organizaram-se os dados em um banco de dados do Programa Microsoft Office Excel 2007 e, em seguida, submeteu-se à análise estatística descritiva e inferencial através do Programa Statistical Package for the Social Sciences (SPSS for Windows), versão 20.0.

Com a finalidade de determinar sua distribuição, as variáveis foram submetidas à análise descritiva e, para verificar a associação entre elas, realizou-se a análise inferencial.

Para organização dos dados, descreveram-se as variáveis como: relacionadas aos dados sociodemográficos; relacionadas à patologia de base, tratamento e adesão à terapia medicamentosa convencional (MC); e relacionadas ao uso de plantas medicinais.

A variável dependente estudada foi o uso de planta medicinal. Já as variáveis independentes estudadas foram aquelas que, possivelmente, tivessem o uso de planta relacionado a elas, quais sejam: nível de atenção, idade, sexo, escolaridade, ocupação, estado civil, renda familiar, patologia de base e adesão à terapia medicamentosa convencional.

O projeto de pesquisa deste estudo recebeu aprovação pelos Comitês de Ética em Pesquisa da Universidade de Fortaleza/UNIFOR (Parecer $n^{\circ}$ 618.827) e do Hospital Universitário Walter Cantídio (Parecer $n^{\circ} 646.419$ ), respeitando os princípios éticos e legais, de acordo com as recomendações da Resolução n. 466/12, do Conselho Nacional de Saúde. 


\section{RESULTADOS}

Dos 210 respondentes da pesquisa, 122 foram considerados válidos para análise. A frequência de distribuição pelo nível de atenção evidenciou que o maior número de participantes, 54,1\% ( $n=66)$, foi representado por aqueles atendidos no nível terciário de atenção (Tabela I).

Tabela I - Distribuição de frequência dos hipertensos e/ou diabéticos participantes da pesquisa $(n=122)$ conforme nível de atenção. Fortaleza, Ceará, Brasil, 2015.

\begin{tabular}{lll}
\hline Variável & $\mathbf{n}$ & \% \\
\hline Nível de atenção & & \\
Terciário (ambulatório especializado) & 66 & 54,1 \\
Primário (UAPS) & 56 & 45,9 \\
\hline
\end{tabular}

UAPS: Unidades de Atenção Primária à Saúde

$\mathrm{Na}$ amostra considerada, obteve-se uma variação de idade entre 23 e 88 anos. A média de idade encontrada foi de $59,46 \pm 12,720$ anos. A faixa etária predominante foi daqueles com 60 anos ou mais $(50,8 \% ; n=62)$. Destes, $70,5 \%(n=86)$ eram do sexo feminino; atingiram, em maior frequência, o nível de escolaridade ensino fundamental incompleto (32\%; $n=39)$; e eram aposentados ou pensionistas $(45,8 \% ; n=55)$. Relativo ao estado civil, $57,6 \%(n=68)$ eram casados ou viviam em união consensual (Tabela II).

Tabela II - Distribuição de frequência dos hipertensos e/ou diabéticos conforme dados sociodemográficos. Fortaleza, Ceará, Brasil, 2015.

\begin{tabular}{|c|c|c|c|c|}
\hline Variáveis & $\mathbf{n}$ & $\%$ & Média & \pm Desvio Padrão \\
\hline Idade (anos completos) ( $n=122)$ & & & 59,46 & 12,720 \\
\hline $20-29$ & 02 & 1,6 & & \\
\hline $30-39$ & 03 & 2,5 & & \\
\hline $40-49$ & 25 & 20,5 & & \\
\hline $50-59$ & 30 & 24,6 & & \\
\hline 60 ou mais & 62 & 50,8 & & \\
\hline \multicolumn{5}{|l|}{ Sexo $(n=122)$} \\
\hline Feminino & 86 & 70,5 & & \\
\hline Masculino & 36 & 29,5 & & \\
\hline \multicolumn{5}{|c|}{ Escolaridade (grau de instrução) $(n=122)$} \\
\hline Analfabeto & 21 & 17,2 & & \\
\hline Alfabetizado & 09 & 7,4 & & \\
\hline Fundamental completo & 17 & 13,9 & & \\
\hline Fundamental incompleto & 39 & 32,0 & & \\
\hline Médio completo & 21 & 17,2 & & \\
\hline Médio incompleto & 03 & 2,5 & & \\
\hline Superior & 12 & 9,8 & & \\
\hline \multicolumn{5}{|l|}{ Ocupação (n=120*) } \\
\hline Sim & 29 & 24,2 & & \\
\hline Não & 36 & 30,0 & & \\
\hline Aposentado/ pensionista & 55 & 45,8 & & \\
\hline \multicolumn{5}{|l|}{ Estado civil $\left(n=118^{\star}\right)$} \\
\hline Casados/ união consensual & 68 & 57,6 & & \\
\hline Solteiros & 21 & 17,8 & & \\
\hline Viúvos & 16 & 13,6 & & \\
\hline Divorciados/ separados & 13 & 11,0 & & \\
\hline
\end{tabular}

Nota: *Foram excluídos da análise aqueles que omitiram suas respostas 
Ao serem questionados se faziam ou já fizeram uso de algum tipo de planta medicinal concomitante ao MC, $62,3 \%(n=76)$ dos participantes da população amostrada responderam que sim. Verificou-se que a ingestão das ervas medicinais não é relatada ao profissional que faz o acompanhamento nas unidades de saúde da família e no ambulatório, uma vez que, neste estudo, dentre aqueles que usaram plantas medicinais para tratar HAS e/ou DM, 78,3\% ( $n=36)$ negaram ter informado ao médico ou enfermeiro sobre a ingestão concomitante ao uso do MC.

Contudo, considerável número de participantes informou ter iniciado uso das plantas medicinais após diagnóstico médico das suas patologias $(76 \%, \mathrm{n}=35)$.

Tabela III - Distribuição de frequência dos hipertensos e/ou diabéticos conforme patologia de base, uso de planta medicinal, finalidade do uso, relato ao médico, início e obtenção. Fortaleza-Ceará, Brasil, 2015.

\begin{tabular}{lll}
\hline Variáveis & $\mathbf{n}$ & $\%$ \\
\hline Patologia de base (HAS/DM) (n=122) & & 40,2 \\
HAS+DM & 49 & 35,2 \\
DM & 43 & 24,6 \\
HAS & 30 & \\
Uso de planta medicinal (n=122) & & 62,3 \\
Sim & 76 & 37,7 \\
Não & 46 & \\
Finalidade do uso (n=76) & & 60,5 \\
Tratar HAS e/ou DM & 46 & 39,5 \\
Outras finalidades & 30 & 21,7 \\
Relato ao médico (n=46) & & 78,3 \\
Sim & 10 & 24,0 \\
Não & 36 & 76,0 \\
Início (n=46) & & \\
Antes do diagnóstico & 11 & 36,9 \\
Depois do diagnóstico & 35 & 19,6 \\
Obtenção (n=46) & & 10,9 \\
Feira livre & 17 & 8,7 \\
Cultivo e produção própria (domicílio) & 09 & 2,2 \\
Produtor ou fornecedor caseiro & 05 & 21,7 \\
Amigo/vizinho/familiar & 04 & \\
Farmácia & 01 & \\
Outros & 10 & \\
\hline
\end{tabular}

HAS: hipertensão arterial sistêmica. DM: diabetes mellitus.

Nota: *Foram excluídos da análise aqueles que omitiram suas respostas

Quanto ao local de obtenção das plantas, a maior parcela dos entrevistados que usaram plantas $(36,9 \%, n=17)$ revelou ter adquirido em feira livre, seguido pela produção domiciliar própria $(19,6 \%, n=9)$. Conforme a tabela IV, para a menor parcela de participantes $(4,4 \%, n=2)$, o alívio de alguns sintomas específicos foi o que se esperou com a ingestão das ervas, sendo referidos nervosismo, tontura, prurido cutâneo e cefaleia.

Concernente à identificação de reações adversas provocadas pelo uso de plantas medicinais, grande parcela relatou não ter identificado $(87 \%, n=40)$. Por fim, quando questionados sobre a suspensão da planta medicinal e do MC, $54,3 \%(n=25)$ disseram não ter suspendido a planta e 100\% $(n=46)$ asseguraram não suspenderem o MC.

Empregou-se a análise inferencial para analisar a associação entre algumas das variáveis independentes e a variável dependente uso de planta medicinal. Foi encontrada associação significativa entre o nível de atenção $(p=0,0001)$, escolaridade $(p=0,021)$, patologia de base $(p=0,0001)$ e a variável de desfecho uso de planta medicinal, no entanto não foi evidenciada associação significativa entre consumo de planta medicinal e idade $(p=0,176)$, sexo $(p=0,070)$, ocupação $(p=0,470)$, estado civil $(p=0,338)$ (Tabela $\vee)$. 
Tabela IV - Distribuição de frequência dos hipertensos e/ou diabéticos conforme ação esperada, reação adversa, suspensão das plantas medicinais e suspensão do medicamento convencional. Fortaleza, Ceará, Brasil, 2015.

\begin{tabular}{llc}
\hline Variáveis & $\mathbf{n}$ & \% \\
\hline Ação esperadas com uso da planta medicinal $(\mathbf{n = 4 6 )}$ & & 56,5 \\
Controle/melhora da resposta do tratamento & 26 & 28,2 \\
Curar HAS e/ou DM & 13 & 6,5 \\
Melhorar a qualidade de vida & 03 & 4,4 \\
Aliviar sintomas & 02 & 4,4 \\
Outros & 02 & 87,0 \\
Reação adversa ( $\mathbf{n}=\mathbf{4 6 )}$ & & 13,0 \\
Não identificaram & 40 & \\
Identificaram & 06 & 54,3 \\
Suspenderam o uso da planta medicinal (n=46) & & 45,7 \\
Não & 25 & 100,0 \\
Sim & 21 & \\
Suspenderam o uso do medicamento convencional (n=46) & & \\
Não & 46 & \\
Sim & 00 & \\
\hline
\end{tabular}

HAS: hipertensão arterial sistêmica. DM: diabetes mellitus

Nota: *Foram excluídos da análise aqueles que omitiram suas respostas

Tabela V - Comparação entre nível de atenção, idade, sexo, escolaridade, ocupação, estado civil, patologia de base e o uso de plantas medicinais. Fortaleza, Ceará, Brasil, 2015.

\begin{tabular}{|c|c|c|c|}
\hline \multirow{2}{*}{ Variáveis } & \multicolumn{2}{|c|}{ Uso de planta medicinal } & \multirow[b]{2}{*}{ p-valor } \\
\hline & $\operatorname{Sim}(\%)$ & Não (\%) & \\
\hline Nível de atenção & & & 0,0001 \\
\hline Terciário (Ambulatório especializado) & $21(31,8)$ & $45(68,2)$ & \\
\hline Primário (UAPS) & $55(98,2)$ & $01(1,8)$ & \\
\hline Idade & & & 0,176 \\
\hline$<60$ anos & $41(68,3)$ & $19(31,7)$ & \\
\hline$\geq 60$ anos & $35(56,5)$ & $27(43,5)$ & \\
\hline Sexo & & & 0,070 \\
\hline Feminino & $58(67,4)$ & $28(32,6)$ & \\
\hline Masculino & $18(50,0)$ & $18(50,0)$ & \\
\hline Escolaridade & & & 0,021 \\
\hline$<5$ anos de estudo & $24(80,0)$ & $06(20,0)$ & \\
\hline$\geq 5$ anos de estudo & $52(56,5)$ & $40(43,5)$ & \\
\hline Ocupação & & & 0,470 \\
\hline Com & $20(69,0)$ & $56(61,5)$ & \\
\hline Sem & $9(31,0)$ & $35(38,5)$ & \\
\hline Estado civil & & & 0,338 \\
\hline Com companheiro & $44(64,7)$ & $24(35,3)$ & \\
\hline Sem companheiro & $28(56,0)$ & $22(44,0)$ & \\
\hline Patologia de base & & & 0,0001 \\
\hline $\mathrm{HAS}+\mathrm{DM}$ & $25(51,0)$ & $24(49,0)$ & \\
\hline DM & $22(51,2)$ & $21(48,8)$ & \\
\hline HAS & $29(96,7)$ & $01(3,3)$ & \\
\hline
\end{tabular}

HAS: hipertensão arterial sistêmica. DM: diabetes mellitus 
Percebe-se que, embora não exista associação significativa entre as variáveis estudadas e o uso de planta medicinal, aspectos importantes foram observados. Em relação à idade, verificou-se que o consumo de planta medicinal foi maior nos participantes menores de 60 anos $(68,3 \%, n=41)$; um predomínio do sexo feminino relacionado ao maior consumo de planta medicinal $(67,4 \%, n=58)$ e que, no sexo masculino, frequência idêntica foi observada entre os que usam $(50 \%, n=18)$ e os que não usam $(50 \%, n=18)$.

Ao investigar a variável ocupação, constatou-se que o maior número de respondentes que ingere planta medicinal tem ocupação $(69 \%, n=20)$. Em relação à variável estado civil, percebeu-se que aqueles com companheiro fazem mais uso de planta medicinal $(64,7 \%, \mathrm{n}=44)$.

\section{DISCUSSÃO}

Este é um dos primeiros estudos acerca do uso de plantas medicinais por pacientes hipertensos e diabéticos em uma população do Nordeste. As regiões Sul e Sudeste possuem mais publicações sobre o tema, em detrimento da escassez de estudos nas regiões Norte e Nordeste do Brasil.

Assim como observado neste estudo, a literatura especializada também identifica elevada resposta positiva $(62,4 \%)$ entre hipertensos, diabéticos e dislipidêmicos ${ }^{(6)}$ Atualmente, as doenças cardiovasculares representam cerca de $40 \%$ das mortes registradas no país, particularmente entre idosos ${ }^{(7)}$.

Em concordância com outro estudo, houve predominância de pessoas do sexo feminino, acima de 60 anos e de baixa escolaridade ${ }^{(8)}$. A associação positiva encontrada entre o uso de remédios caseiros e a idade pode ser explicada, pelo menos em parte, pela característica transmissão de conhecimento popular, que ocorre, principalmente, através das gerações ${ }^{(9)}$.

O fato do consumo de plantas medicinais ser um dos mais antigos meios empregados pela humanidade para tratar, curar e prevenir as doenças ao longo dos tempos ${ }^{(10)}$, associado ao fácil acesso às mesmas ${ }^{(4)}$, parece favorecer a sua utilização por considerável parcela da população.

A literatura especializada revela que as plantas medicinais e seus derivados são amplamente utilizados como terapêutica alternativa, no Brasil, sobretudo por pessoas portadoras de doenças crônicas não transmissíveis (DCNT) e que estão em tratamento com outros medicamentos ${ }^{(11) .}$

Em consonância com este estudo, pesquisadores confirmaram que $76,4 \%$ dos participantes não informaram aos seus oncologistas sobre o tratamento complementar com plantas medicinais e a justificativa apresentada para $o$ fato foi que os médicos nunca haviam feito questionamento a esse respeito ${ }^{(12)}$. A comunicação aos médicos de que estão consumindo plantas medicinais em conjunto com o MC, para 59,4\%, nunca acontece ou somente acontece quando passam mal ${ }^{(10)}$.

Há, ainda, por parte de alguns usuários das plantas medicinais e de seus derivados, a falsa ideia de que tais produtos, por serem naturais, são inócuos e, por isso, não apresentariam riscos a sua saúde, portanto, os profissionais devem estar atentos aos riscos potenciais oferecidos pela associação entre fármacos e plantas medicinais ${ }^{(13)}$.

É conveniente lembrar que o consumo excessivo de algumas ervas pode trazer riscos potenciais à saúde. Quando ingeridas na forma de chá ou "in natura", as plantas podem acarretar efeitos inesperados, tais como: alergias, alterações cardíacas, hormonais, irritativas e purgativas ${ }^{(14)}$. Para tanto, é imprescindível o conhecimento das propriedades terapêuticas, das indicações, da forma de utilização e preparo da planta, a fim de que se conserve o princípio ativo e possam ser garantidas a eficácia e segurança do tratamento.

A possível toxicidade intrínseca à planta, além da contaminação por agentes externos e por metais pesados e pesticidas, são alguns dos riscos quando as plantas são adquiridas em feiras livres, mercados públicos ou lojas de produtos naturais ${ }^{(8)}$.

A maioria dos pacientes ( $>70 \%$ ) afirmou que iniciou o consumo depois de ter a patologia diagnosticada, demonstrando ser essa a principal motivação para a ingestão, semelhante ao relatado na literatura ${ }^{(12)}$. Entretanto, para algumas pessoas, as plantas são utilizadas independentemente do diagnóstico específico de uma patologia, fato comprovado quando $63 \%$ dos entrevistados afirmaram consumir sempre que há alguma indisposição ou problema de saúde ${ }^{(13)}$.

Quanto ao local de obtenção das plantas, a maior parcela dos entrevistados que usou plantas revelou adquirir em feira livre e uma menor parcela tem produção domiciliar própria, opondo-se ao que foi encontrado em outro estudo, que indicou o cultivo domiciliar como sendo o mais prevalente ${ }^{(8)}$.

No presente estudo, um fator que pode ter interferido nesse resultado é a população pesquisada ser essencialmente da zona urbana. O processo de migração da população rural para a zona urbana e a urbanização das cidades 
favorece a perda do conhecimento sobre as plantas medicinais, tanto pela escassez de quintais com jardins como pelo desinteresse das novas gerações no aprendizado acumulado pelos antepassados sobre as propriedades medicinais $^{(13)}$.

Os dados desta pesquisa apontam que mais da metade dos pacientes demonstram que os resultados esperados com a ingestão das plantas medicinais foi osentimento de controle sobre a doença e a melhora da resposta ao tratamento convencional. Em estudo numa comunidade rural do Rio Grande do Sul, foi evidenciado que $75 \%$ referiram sentir melhora nos sintomas após emprego do uso associado à medicação convencional(15).

Para uma pequena parcela de participantes (menos de 5\%), o alívio de alguns sintomas específicos foi o que se esperou com a ingestão das ervas, sendo referidos nervosismo, tontura, prurido cutâneo e cefaleia. A cura foi o que mais se buscou pelos participantes (19\%), seguida pelo alívio de outros sintomas e a melhora da resposta do tratamento convencional(12).

Concernente à identificação de reações adversas provocadas pelo uso de plantas medicinais, grande parcela relatou não ter identificado. Na contramão disso, houve relato de apenas $4 \%$ de reações ou intoxicações relacionadas às ervas em pacientes do estado do Paraná(16). Esse número, no entanto, deveria ser bem mais elevado, dado o considerável aumento no consumo das plantas pela população.

Por fim, quando questionados sobre a suspensão da planta medicinal e do $\mathrm{MC}$, a maioria disse não ter suspendido a planta e todos asseguraram não suspenderem o MC, assemelhando-se a outro estudo, em que $54,7 \%$ dos participantes descontinuaram o uso das plantas medicinais e/ou derivados e nenhum descontinuou o tratamento convencional(12). Esses dados demonstram que, pelo presente estudo, os hipertensos e diabéticos sentem segurança no tratamento alopático, mas também acenam para o fato do consumo de plantas medicinais também ser bem aceito e continuado por eles, evidenciando que a junção das duas terapias, convencional e alternativa, de forma orientada e responsável, poderia trazer inúmeros benefícios para a comunidade.

Em contrapartida, estudo realizado no Sul do país relatou que houve substituição dos medicamentos convencionais por plantas medicinais, de forma que muitos entrevistados afirmaram que achavam que poderiam melhorar os sintomas com remédio caseiro, que trabalhavam e não tinham receita para comprar o medicamento ${ }^{(8)}$.

Estudo publicado em 2014(17), comparou os períodos de 1990-2002 com 2003-2013, constatando que houve um pequeno aumento nas publicações relacionadas ao tema "Plantas medicinais e atenção primária à saúde". Os motivos para a pouca adesão estariam relacionados ao baixo interesse acadêmico no tema, possivelmente devido à falta de incentivo do poder público; o conhecimento ser antigo e sagrado, não fazendo parte de um futuro envolvendo novas tecnologias; e pela integração falha entre as diferentes áreas do conhecimento envolvidas.

Por fim, apesar da utilização pela maior parcela da população pesquisada, ressalta-se que o uso de plantas medicinais não alterou o uso de medicamentos convencionais e que a confiabilidade dos usuários quanto aos efeitos benéficos dessa prática poderiam ser mais explorados pelos profissionais da saúde, afim de explicar possíveis riscos/interações da utilização de algumas plantas em concomitância com outros remédios. Sugere-se que esse tema seja mais abordado durante os cursos de graduação como complemento da grade curricular, haja vista ser uma prática muito constante no Brasil.

\section{CONCLUSÃO}

O uso de plantas medicinais se faz presente na população pesquisada e a elevada prevalência da utilização requer mais estudos acerca dos benefícios e malefícios dessa prática, bem como a capacitação dos profissionais envolvidos, para consequente segurança, eficácia e consumo racional pelos pacientes.

\section{AGRADECIMENTOS}

Ao CNPq, à FINEP e à FUNCAP, pelo incentivo científico e apoio financeiro.

\section{CONFLITOS DE INTERESSE}

Não houve conflitos de interesse no presente estudo.

Este artigo é parte da dissertação intitulada: Utilização de Plantas Medicinais por uma população de hipertensos e diabéticos atendidos no município de Fortaleza, apresentada ao Programa de Mestrado em Saúde Coletiva da Universidade de Fortaleza (UNIFOR), em 2015, contendo 166 páginas. 


\section{REFERÊNCIAS}

1. World Health Organization. Traditional medicine strategy 2002-2005. Geneva: World Health Organization; 2002.

2. Ministério da Saúde (BR), Departamento de Atenção Básica. Estratégias para o cuidado da pessoa com doença crônica: diabetes mellitus. Brasília: Ministério da Saúde; 2013.

3. Rodrigues AG, Santos MG, De Simoni C. Fitoterapia na saúde da família. In: Sociedade Brasileira de Medicina de Família e Comunidade. Programa de Atualização em Medicina de Família e Comunidade (PROMEF). Porto Alegre: Artmed; 2011. p. 131-65.

4. Castro MS, Barros NF, Alegre SM, Hoehne EL. O uso de terapia alternativa e complementar por pacientes diabéticos do tipo 2. Brasília Méd. 2010;47(1):17-25.

5. Nicoletti MA, Oliveira MA Jr, Bertasso CC, Caparossi PY, Tavares APL. Principais interações no uso de medicamentos fitoterápicos. Infarma [Internet]. 2007 [acesso em 2015 Jan 18];19(1-2):32-40. Disponível em: http://www.revistas. cff.org.br/infarma/article/view/222

6. Silva $B Q$, Hahn SR. O uso de plantas medicinais por indivíduos com hipertensão arterial sistêmica, diabetes mellitus ou dislipidemias. R Bras Farm Hosp Serv Saúde [Internet]. 2011 [acesso em 2015 Jan 18];2(3):36-40. Disponível em: http://www.sbrafh.org.br/rbfhss/public/artigos/RBFHSSV2N3\%20artigo07.pdf

7. Piuvezam G, Medeiros WR, Costa AV, Emerenciano FF, Santos RC, Seabra DS. Mortality from cardiovascular diseases in the elderly: Comparative analysis of two five-year periods. Arq Bras Cardiol [Internet]. 2015 [acesso em 2015 Jan 18];105(4):371-80. Disponível em: http://www.scielo.br/pdf/abc/2015nahead/0066-782X-abc-20150096. pdf

8. Zeni ALB, Parisotto AV, Mattos G, Helena ETS. Utilização de plantas medicinais como remédio caseiro na Atenção Primária em Blumenau, Santa Catarina, Brasil. Ciênc Saúde Colet [Internet]. 2017 [acesso em 2015 Jan 11];22(8):2703-2712. Disponível em: http://www.scielo.br/scielo.php?pid=S1413-81232017002802703\&script=sci_ abstract\&tlng=pt

9. Parisius LM, Stock-Schröer B, Berger S, Hermann K, Joos S. Use of home remedies: across-sectional survey of patients in Germany. BMC Fam Pract [Internet]. 2014 [acesso em 2015 Jan 18];15:116. Disponível em: https:// www.ncbi.nlm.nih.gov/pubmed/24916173

10. Veiga VF Jr. Estudo do consumo de plantas medicinais na Região Centro-Norte do Estado do Rio de Janeiro: aceitação pelos profissionais de saúde e modo de uso pela população. Rev Bras Farmacogn [Internet]. 2008 [acesso em 2015 Jan 18];18(2):308-13. Disponível em: http://www.scielo.br/scielo.php?pid=S0102695X2008000200027\&script=sci_abstract\&tIng=pt

11. Alexandre RF, Bagatini $F$, Simões $\mathrm{CMO}$. Interações entre fármacos e medicamentos fitoterápicos à base de ginkgo ou ginseng. Rev Bras Farmacogn [Internet]. 2008 [acesso em 2015 Jan 18];18(1):117-26. Disponível em: http://www.scielo.br/scielo.php?script=sci_arttext\&pid=S0102-695X2008000100021

12. Vieira RCF. Estudo do uso de plantas medicinais e/ou produtos à base de plantas medicinais com o tratamento complementar, por pacientes atendidos no Centro de Pesquisas Oncológicas CEPON/SC [dissertação]. Florianópolis: Universidade Federal de Santa Catarina; 2008.

13. Veiga VF Jr, Pinto AC, Maciel MAM. Plantas medicinais: cura segura? Quím Nova [Internet]. 2005 [acesso em 2015 Jan 18];28(3):519-28. Disponível em: http://www.scielo.br/scielo.php?pid=S0100-40422005000300026\&script=sci_ abstract\&tlng=pt

14. Newall CA, Anderson LA, Phillipson JD. Plantas medicinais: guia para professional de saúde. São Paulo: Editorial Premier; 2002.

15. Piriz MA, Mesquita MK, Cavada CT, Palma JS, Ceolin T, Heck RM. Uso de plantas medicinais: impactos e perspectivas no cuidado de enfermagem em uma comunidade rural. Rev Eletrônica Enferm [Internet]. 2013 [acesso em 2015 Jan 18];15(4):992-9. Disponível em: https://revistas.ufg.br/fen/article/view/19773

16. Viganó J, Viganó JÁ, Cruz-Silva CTA. Utilização de plantas medicinais pela população da região urbana de Três Barras do Paraná. Acta Sci Health Sci [Internet]. 2007 [acesso em 2015 Jan 18];29(1):51-8. Disponível em: http://periodicos.uem.br/ojs/index.php/ActaSciHealthSci/article/view/106 
17. Antonio GD, Tesser CD, Moretti-Pires RO. Phytotherapy in primary health care. Rev Saude Publica [Internet]. 2014 [acesso em 2015 Jan 18];48(3):541-53. Disponível em: https://www.ncbi.nlm.nih.gov/pubmed/25119949

\section{Endereço para correspondência:}

Taís Batista Virgínio

Universidade de Fortaleza - UNIFOR

Programa de Pó-Graduação em Saúde Coletiva

Av. Washington Soares, 1321, Bloco S, Sala 1

Bairro: Edson Queiroz

CEP: 60.811-905 - Fortaleza - CE - Brasil

E-mail: tais_bv@hotmail.com 